\title{
HRTEM OF EXTENDED DEFECTS IN TL-2212 THIN FILMS
}

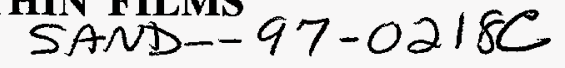

P.P. NEWCOMER*, E. L. VENTURINI*, H. SCHÖNE ${ }^{\Phi}$, B. L. DOYLE*, K. E. MYERS ${ }^{\psi}$

*Sandia National Laboratories, Albuquerque, NM 87185

ФPhillips Laboratory/VTMC, Kirtland AFB, NM 87117

భDupont Superconductivity, Wilmington, DE 19880

\section{ABSTRACT}
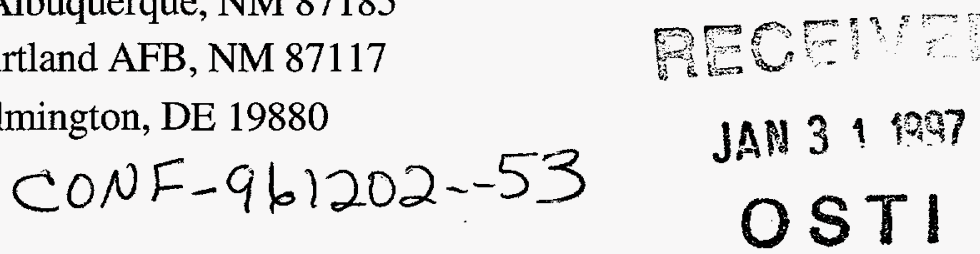

Many applications of high temperature superconductors, HTS, require the presence of lattice defects in the material structure to suppress the motion of magnetic vortices and enhance the critical current density, $\mathrm{J}_{c}$. The microstructure of $\mathrm{Tl}_{2} \mathrm{Ba}_{2} \mathrm{CaCu}_{2} \mathrm{O}_{8-\delta}$ (Tl-2212) thin films which have extended defects induced by high energy $\mathrm{Au}$ and $\mathrm{Cu}$ ion irradiation is studied using high resolution transmission electron microscopy, HRTEM, with slow scan digital imaging. In order to optimize the HTS properties and better analyze the consequent microstructural modification, the fluence is varied. At moderate fluences, resulting in $\sim 4 \%$ reduction of the superconducting transition, large enhancements of $J_{c}$ and vortex pinning potential are observed. The density and microstructure of isolated defects and surrounding structure will be discussed and compared to damage profiles calculated using the TRIM code. Correlation will be made between the HRTEM results and the changes in HTS properties.

\section{INTRODUCTION}

Many applications of high-temperature superconductors, HTS, require large critical current densities, $\mathrm{J}_{\mathrm{c}}$, at superconducting temperature in high magnetic fields, B. Under these conditions magnetic vortices penetrate the HTS and experience strong Lorentz forces which cause motion of the vortices and therefore dissipation [1]. Early work has shown some improvement in vortex pinning at localized structural defects induced by neutron or ion irradiation $[2,3]$. Significant enhancement in $\mathrm{J}_{c}$ and vortex pinning was shown in a $\mathrm{Tl}_{2} \mathrm{Ba}_{2} \mathrm{Ca}_{2} \mathrm{Cu}_{3} \mathrm{O}_{10-\delta}, \mathrm{Tl}-2223$, single crystal after irradiation with $4.5 \mathrm{MeV}$ protons. The superconducting transition temperature, $\mathrm{T}_{c}$, decreased, but the $\mathrm{J}_{\mathrm{c}}$ increased by an order of magnitude [4]. Irradiation can enhance $\mathrm{J}_{\mathrm{c}}$ at low fluences; however, as fluence is increased $\mathrm{T}_{\mathrm{c}}$ decreases and an increase normal state resistivity results $[5,6]$. Considerably less improvement was observed in $\mathrm{Tl}_{2} \mathrm{Ba}_{2} \mathrm{CaCu}_{2} \mathrm{O}_{8-\delta}, \mathrm{Tl}-2212$, films for $2 \mathrm{MeV}$ protons [7]. A microstructural study with HRTEM showed damage by $1.5 \mathrm{MeV} \mathrm{Kr}$ ions in Tl2212 single crystals consisted of clustered point defects in localized amorphized regions [8].

Strong vortex pinning in $\mathrm{YBa}_{2} \mathrm{Cu}_{3} \mathrm{O}_{7-\mathrm{x}}, \mathrm{YBCO}$, single crystals after high energy heavy ion irradiation was observed $[9,10]$. HRTEM has shown that pinning sites are amorphous, linear damage tracks [11]. Tl-based HTS ceramics [12, 13] and thin films [14, 15] showed strongly enhanced pinning when linear damage tracks were incorporated. Recent work has shown that an intermediate energy ion irradiation, $60 \mathrm{MeV} \mathrm{Cu}$, in Tl-2212 thin films resulted in extended defects which greatly enhanced the vortex pinning and $J_{c}[16]$.

The goal of this study is to induce the formation of damage tracks in Tl-2212 thin films at ion velocities which are widely available at accelerator facilities. Ion irradiations, produced by a Tandem Van de Graaff accelerator at the Sandia Ion Beam Research Facility, at $60 \mathrm{MeV} \mathrm{Cu}^{+9}$ and $88 \mathrm{MeV}$ Au on Tl-2212 films are compared.

\section{EXPERIMENTAL METHODS}

Thin films of Tl-2212, approximately $600 \mathrm{~nm}$ thick, were grown by a two step process. Ba-Ca-Cu-O precursor film was deposited onto an unheated $\mathrm{LaAlO}_{3}$ single crystal substrate by offaxis planar magnetron sputtering [17]. An anneal at $850 \mathrm{C}$ in the presence of $\mathrm{Tl}-\mathrm{Ba}-\mathrm{Ca}-\mathrm{Cu}-\mathrm{O}$ and $\mathrm{Tl}_{2} \mathrm{O}_{3}$ powders thallinates the film. These films typically exhibit strong $c$-axis normal orientation and are highly oriented in the plane as well. The films have superconducting onsets greater than $100 \mathrm{~K}$. The films have low microwave surface resistance, and high $\mathrm{J}_{\mathrm{c}}$ 's of $2 \times 10^{6} \mathrm{~A} / \mathrm{cm}^{2}$ at $77 \mathrm{~K}$ [18].
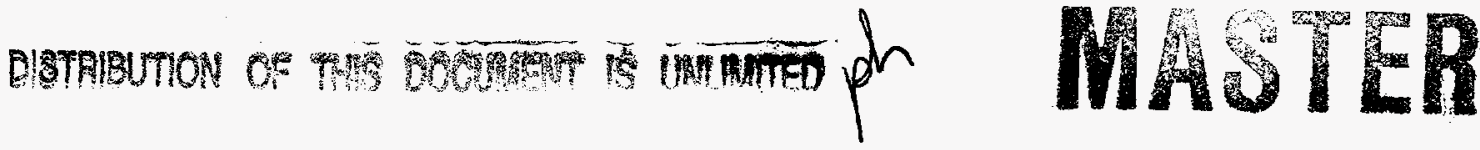


\section{DISCLAIMER}

This report was prepared as an account of work sponsored by an agency of the United States Government. Neither the United States Government nor any agency thereof, nor any of their employees, make any warranty, express or implied, or assumes any legal liability or responsibility for the accuracy, completeness, or usefulness of any information, apparatus, product, or process disclosed, or represents that its use would not infringe privately owned rights. Reference herein to any specific commercial product, process, or service by trade name, trademark, manufacturer, or otherwise does not necessarily constitute or imply its endorsement, recommendation, or favoring by the United States Government or any agency thereof. The views and opinions of authors expressed herein do not necessarily state or reflect those of the United States Government or any agency thereof. 


\section{DISCLAIMER}

Portions of this document may be illegible in electronic image products. Images are produced from the best available original document. 
The Tl-2212 thin films were cleaved into $3 \times 3 \mathrm{~mm}$ samples and were irradiated with 60 $\mathrm{MeV} \mathrm{Cu}$ to a fluence of $2 \mathrm{X} 10^{11}$ ions $/ \mathrm{cm}^{2}[16]$ and $88 \mathrm{MeV} \mathrm{Au}$ ions $/ \mathrm{cm}^{2}$ at four different fluences ranging from 0.25 to $2.0 \times 10^{11}$ ions $/ \mathrm{cm}^{2}$ using the Sandia Tandem Accelerator. The incident ion direction was slightly off the normal to the film surface (the crystallographic $c$-axis). The low dose rate of $7 \times 10^{8}$ ions $/ \mathrm{sec} / \mathrm{cm}^{2}$ causes no heating during irradiation. The ion stopping power or energy loss, $d E / d x$, in Tl-2212 was calculated using a Monte-Carlo based simulation code, TRIM [19]. TRIM does not model track formation, but the estimated $d E / d x$ for these incident ions in Tl-2212 was compared to detailed damage morphology and stopping power studies of irradiated yttrium iron garnets $[20,21,22]$.

HRTEM was done on the JEOL 2010 equipped with a Gatan 694 slow-scan camera in the Earth and Planetary Sciences Department at University of New Mexico using phase contrast imaging with the (100) diffracting beams. The data were analyzed quantitatively using the Gatan's Digital Micrograph software. Superconducting properties were measured with a Quantum Design MPMS SQUID magnetometer.

\section{RESULTS}

Using the yttrium iron garnet studies $[20,21,22]$ as a guide, we estimated an ion stopping power of more than $4.5 \mathrm{keV} / \mathrm{nm}$ is needed to form elongated or extended defects. Approximately $20 \mathrm{keV} / \mathrm{nm}$ is needed to produce continuous cylindrical linear damage tracks in a complex oxide. TRIM calculations show $d E / d x$ of $12.5 \mathrm{keV} / \mathrm{nm}$ for $60 \mathrm{MeV} \mathrm{Cu}$ and $19.5 \mathrm{keV} / \mathrm{nm}$ for $88 \mathrm{MeV} \mathrm{Au}$ in T1-2212 using a crystallographically determined density and the TRIM default displacement energies. Electronic and nuclear interactions between ion and target atoms are taken into account. The calculated $d E / d x$ of $12.5 \mathrm{keV} / \mathrm{nm}$ for $60 \mathrm{MeV} \mathrm{Cu}$ is below that for linear track formation. HRTEM observation of cross-sectioned irradiated films confirmed extended defects with shapes varying from continuous to discontinuous cylinders. The calculated $d E / d x$ value of $19.5 \mathrm{keV} / \mathrm{nm}$ for $88 \mathrm{MeV} \mathrm{Au}$ is near the $20 \mathrm{keV} / \mathrm{nm}$ threshold for forming linear tracks. Cross-section HRTEM of the resulting damage morphologies show continuous cylindrical extended damage. The amorphous center of the track is believed to originate from a Coulomb explosion after an incident energetic heavy ion leaves a highly ionized column in the material. The radii of the resulting damage columns should be approximately $3.5 \mathrm{~nm}$ for $60 \mathrm{MeV} \mathrm{Cu}$ and $5.0 \mathrm{~nm}$ for $88 \mathrm{MeV}$ Au based on the calculated energy loss rates and the data on yttrium iron garnet. This is in agreement with the observed track radii in this study as shown in figure 1 . The dark rim around each track is a region of recrystallization as indicated by the dark fringes which are slighly out of alignment with the bulk crystal fringes. This region is included in the track measurement.

The number of amorphous regions from the plan-view HRTEM images of the $60 \mathrm{MeV} \mathrm{Cu}$ and $88 \mathrm{MeV} \mathrm{Au}$ irradiated samples were measured and the number of defects per area was calculated. The $60 \mathrm{MeV} \mathrm{Cu}$ irradiation appears to form an amorphous core defect for approximately $90 \%$ of incident ion events while the $88 \mathrm{MeV} \mathrm{Au}$ irradiation appear to have one amorphous core defect per incident ion event.

HRTEM comparison of the $88 \mathrm{MeV} \mathrm{Au}$ to the $60 \mathrm{MeV} \mathrm{Cu}$ irradiated samples indicates that more structural damage occurred during the $\mathrm{Au}$ irradiations at similar fluences. Dark-contrast strain lobes were observed in HRTEM images around the $88 \mathrm{MeV} \mathrm{Au}$ induced tracks as seen in figure 2. There is some light-contrast variation around the $\mathrm{Cu}$ induced amorphous columns which indicates some point defects may exist from secondary recoils, but strain was not observed. TRIM calculations indicate about one target recoil ion/Ångstrom per incident Au ion with energies of $\sim 100$ to $200 \mathrm{eV}$. The energy transferred to these recoil ions constitutes only about 1 to $2 \%$ fraction of the total primary $\mathrm{Au}$ ion energy loss. Target displacement events outside the amorphized core are highly significant because nuclear stopping dominates the recoil ions and because of the high number of recoils produced. For $\mathrm{Cu}$; however, we find that only 0.05 target recoil ion/Ångstrom per incident $\mathrm{Cu}$ are created with energies of $\sim 50$ to $100 \mathrm{eV}$. The absence of a strain region around the core of the $\mathrm{Cu}$ induced tracks compared to the strained $\mathrm{Au}$ induced tracks is expected due to the reduced number of recoils and lower energy transfer.

The total diameter of the damage affected region for the $88 \mathrm{MeV}$ Au induced tracks is about 15 to $25 \mathrm{~nm}$. The HRTEM image of figure 1 does not show the surrounding damage because the lattice fringes reflect the overall periodicity of the structure and do not reveal the point defects. A 
series of increasing fluence was done for the $88 \mathrm{MeV} \mathrm{Au}$ ions in four Tl-2212 films. The images in figure 2 show the increased damage to the originally homogeneous films. At the higher fluences the damaged regions overlap because $2 \times 10^{11}$ defects $/ \mathrm{cm}^{2}$ corresponds to an average separation of $23 \mathrm{~nm}$. For the 2.5 and $5.0 \times 10^{10}$ ions $/ \mathrm{cm}^{2}$ fluences in samples irradiated with $88 \mathrm{MeV} \mathrm{Au}$ ions, isolated damage tracks were observed.
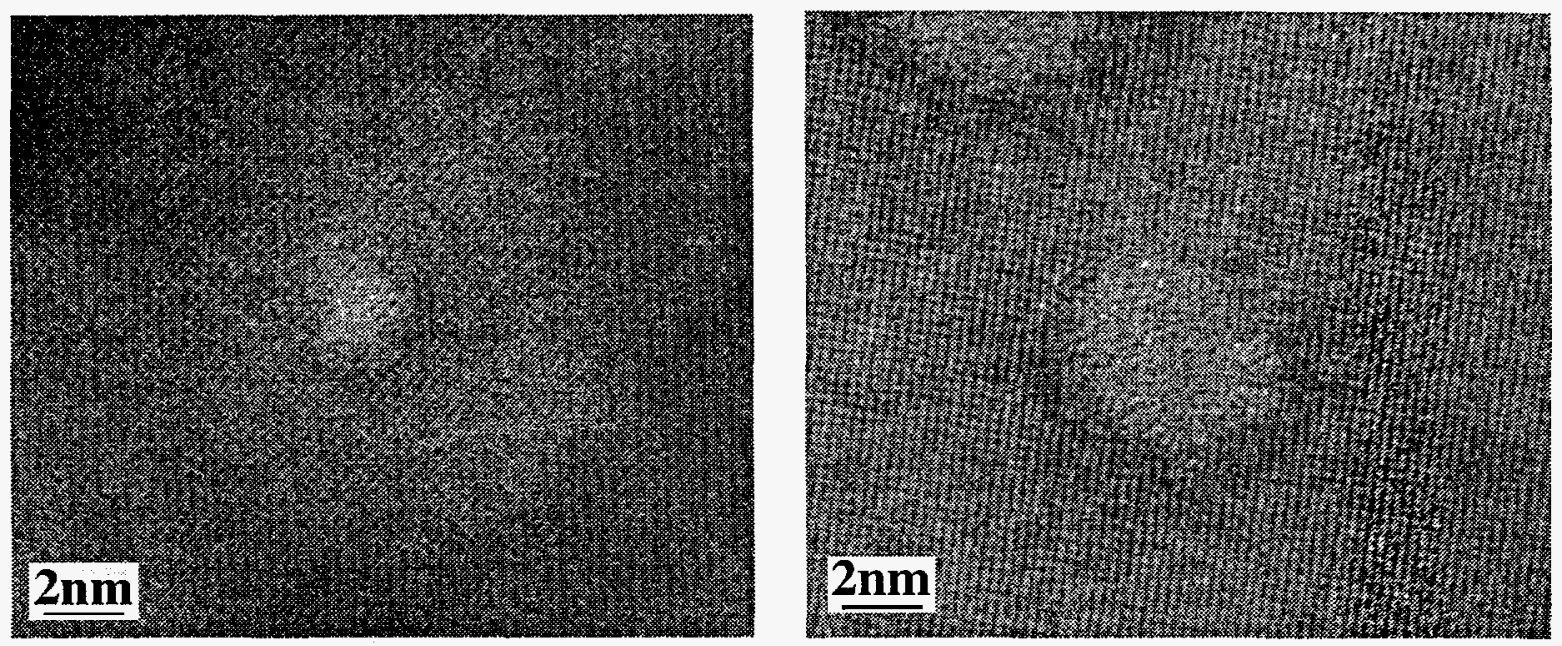

Figure $1 \mathrm{HRTEM}$ plan-view of $60 \mathrm{MeV} \mathrm{Cu}$ (left) and $88 \mathrm{MeV} \mathrm{Au} \mathrm{(right)} \mathrm{irradiation} \mathrm{damage.}$

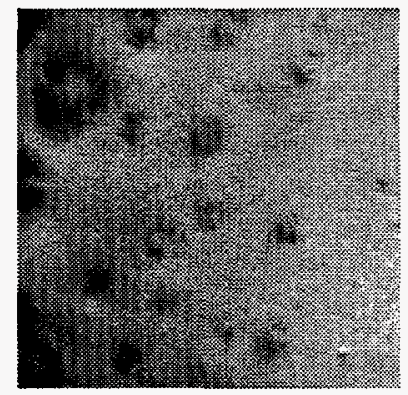

$2.5 \times 10^{10} \mathrm{ion} / \mathrm{cm}^{2}$

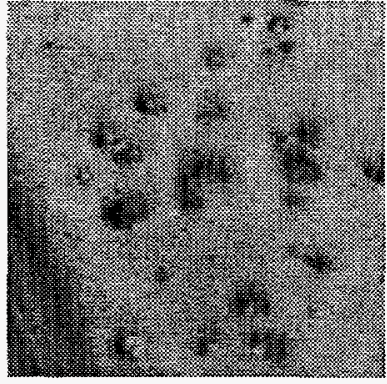

$5 \times 10^{10} \mathrm{ion} / \mathrm{cm}^{2}$

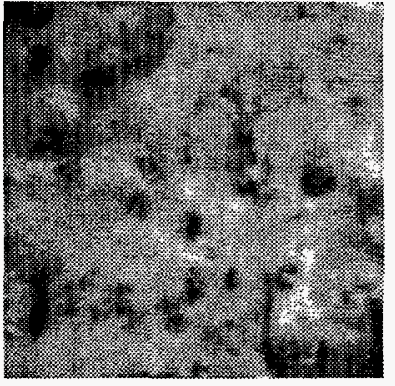

$1 \mathrm{X} 10^{11} \mathrm{ion} / \mathrm{cm}^{2}$

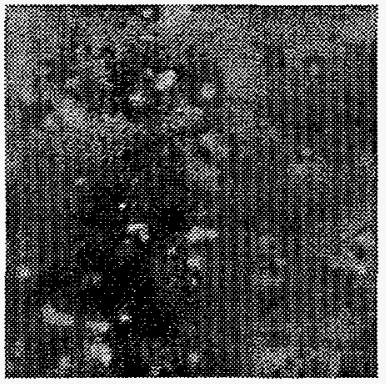

$2 \times 10^{11} \mathrm{ion} / \mathrm{cm}^{2}$

Figure 2 Plan-view TEM after a series of $88 \mathrm{MeV}$ Au fluences on 4 Tl-2212 films. (20nm)

The onset of superconductivity, $T_{c}$, was determined from the Meissner transition (field cooling) in a $0.2 \mathrm{mT}$ field applied normal to the film (along the crystallographic c-axis). Figure 3 compares the high-temperature portion of the transition in an as-grown Tl-2212 film and a series of 4 films irradiated to fluences between 0.25 and $2.0 \times 10^{11}$ ions $/ \mathrm{cm}^{2} 88 \mathrm{MeV} \mathrm{Au}$ irradiation. $\mathrm{T}_{c}$ is decreased by $3 \mathrm{~K}$ (from 101 to $98 \mathrm{~K}$ ) by the lowest fluence irradiation, but the width of the transition is unchanged. At higher fluences, the transition is increasingly broadened.

The effectiveness of extended defects as vortex pinning sites is shown by comparing the magnetic critical current densities, $\mathrm{J}_{\mathrm{c}}$, in the Tl-2212 films before and after irradiation. The semi-log plot in Figure 4 compares $J_{c}$ at $40 \mathrm{~K}$ versus magnetic field applied normal to the Tl-2212 film surface for an as-grown film and films irradiated with $2.5 \times 10^{10}$ to $5 \times 10^{10} \mathrm{Au}$ ions $/ \mathrm{cm}^{2}$ and $2.0 \times 10^{11} \mathrm{Cu}$ ions $/ \mathrm{cm}^{2}$. J was extracted from the isothermal hysteresis loops using the Bean critical state model and the dimensions of the entire sample with the appropriate geometric correction [23]. The relatively weak vortex pinning in the as-grown film is reflected in the rapid decrease in $\mathbf{J}_{c}$ with increasing field; $J_{c}$ drops by nearly three orders of magnitude in a field of only 0.2 tesla. In contrast, 
the extended defects resulting from the $\mathrm{Cu}$ and $\mathrm{Au}$ irradiations provide strong pinning sites for the vortices; $J_{c}$ decreases by only an order of magnitude in a field of 1 tesla following the $\mathrm{Cu}$ irradiation and two orders of magnitude after $\mathrm{Au}$ irradiation. The $\mathrm{Au}$ irradiation at a fluence of $5 \times 10^{10} \mathrm{Au}$ ions $/ \mathrm{cm}^{2}$ produced improvement similar to the dose at $2.5 \times 10^{10}$ ions $/ \mathrm{cm}^{2}$. At even higher $\mathrm{Au}$ fluences less improvement was found, presumably due to the large suppression of $T_{c}$ and the extensive damage from secondary ions.

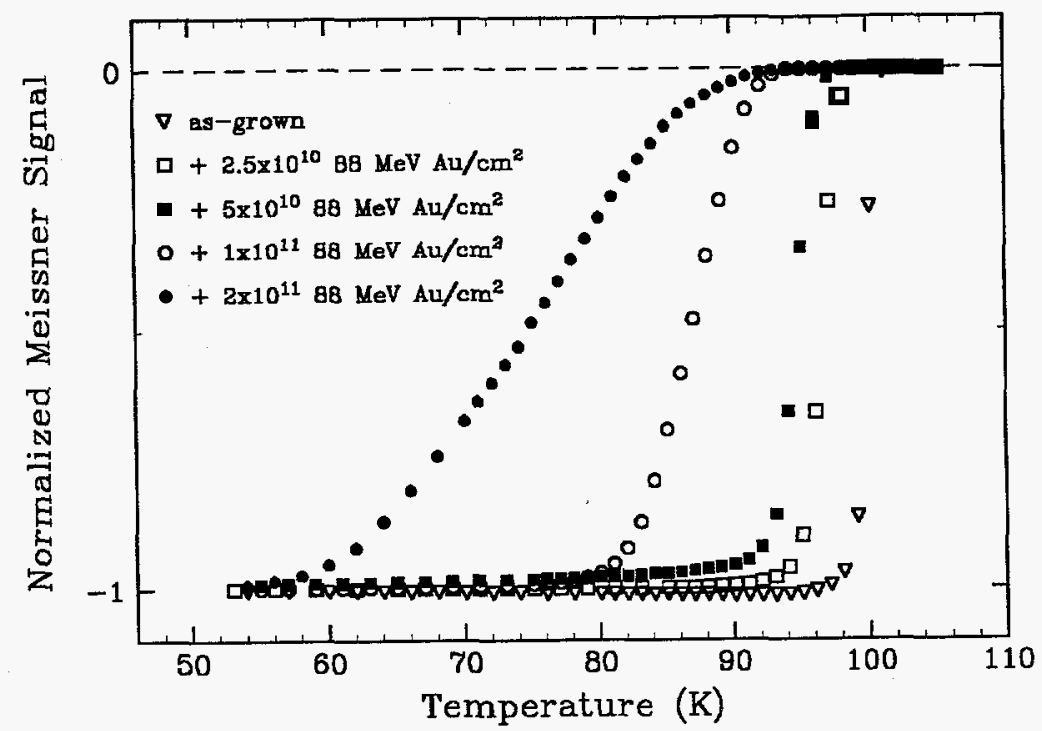

Figure 3. Meissner transition in $0.2 \mathrm{mT}$ (field cooling) for as-grown and irradiated Tl-2212 thin films.

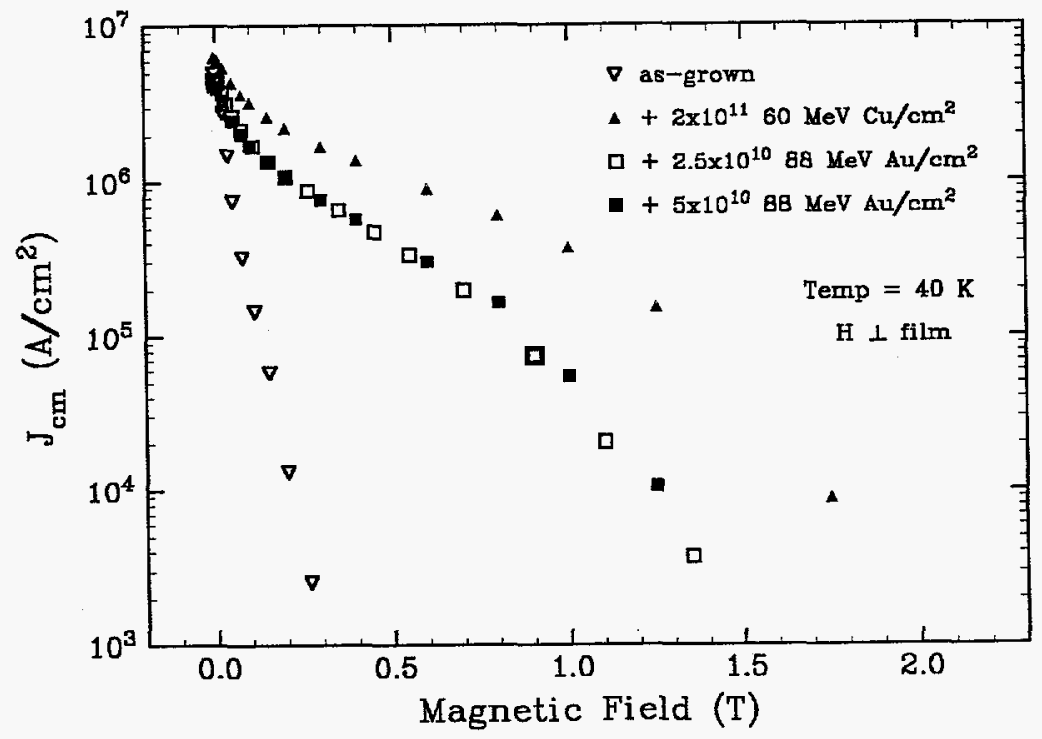

Figure 4. Critical current density $\mathrm{J}_{\mathrm{c}}$ at $40 \mathrm{~K}$ versus applied magnetic field for as-grown and irradiated Tl-2212 films. 


\section{DISCUSSION}

TRIM alone can not predict track formation, as this would require the calculation of time dependent electro-mechanical forces of more than $10^{6}$ ionized target atoms for each incident ion. The damage morphology resulting from the different stopping power regimes has been categorized in previous studies $[20,24]$ for yttrium iron garnet. Using the yttrium iron garnet results as a guide, we determined that we need a stopping power of approximately $20 \mathrm{keV} / \mathrm{nm}$ to produce contiguous tracks. We calculated an energy loss of $12.5 \mathrm{keV} / \mathrm{nm}$ for $60 \mathrm{MeV} \mathrm{Cu}$ and an energy loss of $19.5 \mathrm{keV} / \mathrm{nm}$ for $88 \mathrm{MeV} \mathrm{Au}$. The decrease in the stopping power over the film thickness is less than $7 \%$ in both cases.

The radial and longitudinal damage profile along a heavy ion track depends strongly on $d E / d x$ in the sample. The rate of energy loss is proportional to $Z^{2}$ (atomic number). The velocity dependence of the incident ions on $d E / d x$ can be summarized as follows. For very small ion velocities $\mathrm{v}$ of a few keV/amu (or more accurately for $\mathrm{v}<<\mathrm{v}_{0} ; \mathrm{v}_{0}=$ Bohr velocity) nuclear collisions dominate $d E / d x$, creating a non-contiguous path of randomly displaced target ions. At a higher velocity of $0.1 \mathrm{v}_{0}$ to $\mathrm{Z}^{2 / 3} \mathrm{v}_{0}$, nuclear stopping power will become insignificant and decreases with $1 / \mathrm{v}^{2}$ while the electronic stopping power increases proportional to $\mathrm{v}$. The maximum electronic stopping power will occur for heavy ions at $\sim 1.5$ to $2 \mathrm{MeV} / \mathrm{amu}$. Finally, at ion velocities much larger than $\mathrm{Z}^{2 / 3} \mathrm{v}_{0}$, or velocities of more than $\sim 10 \mathrm{MeV} / \mathrm{amu}$, the electronic stopping power decreases again at a rate of $\ln \left(\mathrm{v}^{2}\right) / \mathrm{v}^{2}$.

There is a low and a high velocity regime were $d E / d x$ can have the same value but differing attributes. Obviously, at low velocity the range of the ions is greatly reduced; however, this does not post any problems for the $600 \mathrm{~nm}$ thick films used in this study. Secondly, and less obvious, is an increase in the track radius over the high velocity track at the same $d E / d x$. This can be easily explained by the increase in the impact parameter dependent ionization cross section of the target atoms as predicted in the Plain-Wave-Born approximation. At the same time, the average energy transferred to the electrons is reduced. This effect has been seen in previous studies [22].

The previous study of yttrium iron garnets found increasingly larger spherical clusters of defects above a stopping power threshold of $4.5 \mathrm{keV} / \mathrm{nm}$ [22]. These defect clusters became elongated and finally formed contiguous cylinders as the stopping power exceeded $20 \mathrm{keV} / \mathrm{nm}$. At this energy loss rate the core of the damage track is amorphous with track radii increasing proportionally with $d E / d x$, as was found in this present study. Using the yttrium iron garnet study as guide, the calculated energy losses of 12.5 and $19.5 \mathrm{keV} / \mathrm{nm}$ should result in tracks of 3.5 and $5 \mathrm{~nm}$, respectively. This is in good agreement with the HRTEM observed data.

HRTEM confirms the damage observed in the Tl-2212 thin films by $60 \mathrm{MeV} \mathrm{Cu}$ are extended (not all columnar) amorphous isolated regions about $3 \mathrm{~nm}$ in diameter with possible point defects surrounding the amorphous core resulting in about $9 \mathrm{~nm}$ total diameter. The damage in Tl2212 films irradiated with $88 \mathrm{MeV} \mathrm{Au}$ are continuous columnar amorphous cores about $5 \mathrm{~nm}$ in diameter with high defect density surrounding the core resulting in about $15 \mathrm{~nm}$ to $25 \mathrm{~nm}$ diameter strained damage regions. Although the Au irradiations resulted in more linear and continuous damage, the $\mathrm{Cu}$ resulted in more enhanced $\mathrm{J}_{c}$ 's. These isolated damage tracks are of a size and density which corresponds to an enhancement in $J_{c}$ 's [1]. The strain surrounding the $\mathrm{Au}$ irradiation induced damage is the result of a higher density of secondary recoil damage. The screening supercurrents in the Meissner state flow through the superoonducting regions between the amorphized extended defects, so the suppression of $T_{c}$ and the broadened transition suggest damage throughout the film from secondary ions generated by collisions with the incident $\mathrm{Au}$ ions. This secondary damage is presumably nonuniform, resulting in the observed broadening.

\section{SUMMARY}

One of the goals of this study was to achieve track formation in Tl-2212 at ion velocities that are obtainable at most accelerator facilities. We have shown that extended defects are created with $60 \mathrm{MeV} \mathrm{Cu}$ and $88 \mathrm{MeV} \mathrm{Au}$ irradiations in Tl-2212. Although not all extended damage from the $\mathrm{Cu}$ irradiation resulted in continuous linear tracks, the enhancement of the pinning was larger than that achieved with continuous linear tracks from the $88 \mathrm{MeV} \mathrm{Au}$ ion irradiation. It appears 
that the much greater strain resulting in larger damage regions accompanying the Au irradiation severely limits the improvement in $\mathrm{J}_{\mathrm{c}}$.

\section{ACKNOWLEDGMENT}

This work was supported by Division of Material Sciences, Office of Basic Energy Sciences, US Department of Energy under Contract DE-AC04-94AL85000. Sandia is a multiprogram laboratory operated by Sandia Corporation, a Lockheed Martin Company, for the US Department of Energy.

\section{REFERENCES}

1. Y. Yeshurun, A.P. Malozemoff, Phys. Rev. Lett. 60 (1988) 2202.

2. A. Umezawa, G. W. Crabtree, J.Z. Liu, H. W. Weber, W.K. Kwok, L.H. Nunez, T.J. Moran, C.H. Sowers, H. Claus, Phys. Rev. B36 (1987) 7151.

3. R.B. van Dover, E.M. Gyorgy, L.F. Schneemeyer, J.W. Mitchell, K.V. Rao, R. Puzniak, J.V. Wasczczak, Nature 342 (1989) 55.

4. J.C. Barbour, E.L. Venturini, D.S. Ginley, Nucl. Instr. \& Meth B59/60, 1395 (1991).

5. J.C. Barbour, E.L. Venturini, D.S. Ginley, and J.F. Kwak, Nucl. Instr. \& Meth. B65, 531 (1992).

6. E.L. Venturini, J.C. Barbour, D.S. Ginley, R.S. Baughman, B. Morosin, Appl. Phys. Lett. 56 (1990) 2456.

7. B.D. Weaver, M.E. Reeves, G.P. Summers, R.J. Soulen, W.L. Olson, M.M. Eddy, T.W. James, E.J. Smith, Appl. Phys. Lett. 59 (1991) 2600.

8. P.P. Newcomer, J.C. Barbour, L.M. Wang, E.L. Venturini, J.F. Kwak, R.C. Ewing, M.L. Miller, B. Morosin, Physica C 267 (1996) 243.

9. L. Civale; A.D. Marwick, T.K. Worthington, M.A. Kirk, J.R., Thompson, L. Krusin-Elbaum, J. Sun, J.R. Clem, F. Holtzberg, Phys. Rev. Lett. 67 (1991)648.

10. M. Konczykowski, F. Rullier-Albenque, E.R. Jacoby, A. Shaulov, J. Yeshurun, P. Lejay, Phys. Rev. B44 (1991) 7167.

11. Y. Zhu, Z.X. Cai, R.C. Budhani, M. Suenaga, D.O. Welch, Phys. Rev. B48 (1993) 6436.

12. V. Hardy, D. Groult, J. Provost, M. Hervieu, B. Raveau, S. Bouffard, Phycia C 178 (1991) 255.

13. A. Wahl, M. Hervieu, G. Van Tendeloo, V. Hardy, J. Provost, D. Groult, Ch. Simon, B.Raveau, Radiation Effects and Defects in Solids 133 (1995) 293.

14. R.C. Budhani, W.L. Holstein, M. Suenaga, Phys. Rev. Lett. 72 (1994) 566.

15. R.C. Budhani, M. Suenaga, S.H. Liou, Phys. Rev. Lett. 69(26) (1992) 3816.

16. E.L. Venturini, P.P. Newcomer, H. Schöne, B.L. Doyle, K.E. Myers, Proceedings of the 1996 IBMM (1996).

17. W.L. Holstein, C. Wilker, D.B. Laubacher, D.W. Face, P. Pang, M.S. Warrington, C.F. Carter, L.A. Parisi, J. Appl. Phys. 74 (1993) 1426.

18. A. Lauder, C. Wilker, D.J. Kountz, W.L. Holstein, D.W. Face, IEEE Trans. Appl. Supercond. 3 (1993) 1683.

19. J. Biersack, L. Haggmark, Nucl. Instr. And Meth. Vol. 174 (1980) andJ. Ziegler, Vol.2-6 Pergamon Press, $1977-1980$.

20. C. Houpert, F. Studer, D. Groult, M. Toulemonde, Nucl. Instrum. Methods B39 (1989) 720.

21. F. Studer and M. Toulemonde, Nucl. Instr. and Meth. B65 (1992) 560.

22. A. Meftah, F. Brisard, J.M. Constantini, M. Hage-Ali, J.P. Stoquert, F. Studer, M. Toulemonde, Phys. Rev. B48 (1993) 920.

23. E.M. Gyorgy, R.B. van Dover, K.A. Jackson, L.F. Schneemeyer and J.V. Waszcrak, Appl. Phys. Lett. 55 (1989) 283.

24. J. Provost, Ch. Simon, M. Hervieu, D. Groult, V. Hardy, F. Studer, M. Toulemonde, MRS Bulletin (1995) 22. 